\title{
To Obtain the OWA Weighting Vector Via Normal Distribution Function
}

\section{Zhiwei LI}

School of Mathematics and Computer Science, Quanzhou Normal University, Quanzhou, China

Xiyang YANG

School of Mathematics and Computer Science, Quanzhou Normal University, Quanzhou, China

Key Laboratory of Intelligent Computing and Information Processing, Fujian Province University, Quanzhou, China.

\begin{abstract}
The OWA operator is an important assessment method in multiple-attribute decision making. A new model based on the density function of normal distribution is given to assign reasonable weights of OWA operators. Using the orness level as a parameter in this method, one can deduce OWA weights by solving a quadratic programing problem. Three propositions of this new model are proven, and a numerical example about the assessment of red wines is given to analyze and illustrate this method.
\end{abstract}

KEYWORD: OWA operators; normal distribution; weighting vector

\section{INTRODUCTION}

The ordered weighted averaging (OWA) operator proposed by Yaer [1] is an important averaging method in multiple-attribute decision making areas such as effectiveness evaluation, neural networks, fuzzy logic controllers [2][3]. Weighting vector is one of the most important issues in an OWA assessment, and the value of a weighting vector is often affected by the degree of optimism of an evaluator.

Though some simple weighing vectors, such as maximum weights; minimum weights and trimmed weights have many practical usages in real life, they cannot meet an evaluator's need when he want to emphasizes the importance of any specific median value; and they cannot be adjusted with the change of the evaluator's degree of optimism. Some modelbased solution partially solve the problems mentioned above. For example, the maximum entropy [4][5], the minimum variability [6], least square deviation [7], minimax disparity approach [8][9], and so on [10].

In [11], we proposed a model based on combination number to obtain a kind of weighting vector. In this paper, a similar model to obtain the OWA weights based on the normal distribution function is proposed.

The following paper is organized as follow. In section 2, the OWA operators and some models of OWA models are introduced. In section 3, a new model is given, and 3 properties of this model are proofed. And finally in section 4, a numerical example about wine assessment is given to show that our new model can reflect the degree of optimism fairly well.

\section{OWA OPERATORS AND MODELS}

\subsection{OWA Operators}

A $n$ dimensional OWA operator with a weighting vector $W=\left(w_{1}, w_{2}, \ldots, w_{n}\right), w_{1}+w_{2}+\cdots+w_{n}=1$, $0 \leq w_{i} \leq 1$, is a function $F_{W}: R^{n} \rightarrow R$, such that[1]

$$
F_{W}\left(x_{1}, x_{2}, \cdots, x_{n}\right)=\sum_{i=1}^{n} w_{i} y_{i},
$$

where $y_{i}$ is the $i$-th largest element in $\left(x_{1}, \cdots, x_{n}\right)$.

For a weighting vector $W=\left(w_{1}, w_{2}, \ldots, w_{n}\right)$, the degree of orness level $\alpha$ is defined as:

$$
\operatorname{orness}(W)=\alpha(W)=\sum_{i=1}^{n} \frac{n-i}{n-1} w_{i},
$$

It is obvious that $\alpha \in[0,1]$. And the degree of optimism of an evaluator can be expressed by the orness level. For example, for a weighting vector $W_{\max }=(1,0, \ldots, 0)$, where an optimistic evaluator consider only the best value in all the attributes, $\alpha\left(W_{\max }\right)=1$. And for another weighting vector $W_{\min }=(0, \ldots, 0,1)$, where the worst value is the only one that a pessimistic evaluator cares, $\alpha\left(W_{\text {min }}\right)=0$. When the evaluator prefers the mean 
of $\left(x_{1}, \cdots, x_{n}\right)$, where $W_{\text {mean }}=(1 / n, 1 / n, \ldots, 1 / n)$ and every value is considered equally important, $\alpha\left(W_{\text {mean }}\right)=1 / 2$.

\subsection{Models to obtain weighting vector}

Besides $W_{\max }, W_{\min }$ and $W_{\text {mean }}$ mentioned above, trimmed mean is another weighting vector that is frequently used. For example, it is used in the assessment of diving competition in 2012 London Olympic Games. Regardless of the largest $m$ numbers and smallest $m$ numbers, trimmed mean averages the rest of the values, thus corresponds to a weighting vector like

$$
W_{\text {trim }}=\left(0, \cdots, 0, \frac{1}{n-2 m}, \cdots, \frac{1}{n-2 m}, 0, \cdots, 0\right)
$$

Different scholars proposed different models to generate weighting vectors. For example, the least square deviation model [7]:

$$
\left\{\begin{array}{c}
\min =\sum_{i=1}^{n-1}\left(w_{i}-w_{i-1}\right)^{2} \\
\text { s.t. } \sum_{i=1}^{n} \frac{n-i}{n-1} w_{i}=\alpha \\
\sum_{i=1}^{n} w_{i}=1 \\
w_{i} \geq 0, i=1,2, \cdots, n
\end{array}\right.
$$

and combination number based OWA model [11]:

$$
\left\{\begin{array}{c}
\min =\sum_{i=1}^{n}\left(w_{i}-\frac{C_{n-1}^{i-1}}{2^{n-1}}\right)^{2}, \\
\text { s.t. } \sum_{i=1}^{n} \frac{n-i}{n-1} w_{i}=\alpha, \\
\sum_{i=1}^{n} w_{i}=1, \\
w_{i} \geq 0, ?=1,2, \cdots, n,
\end{array}\right.
$$

\section{A NEW MODEL TO OBTAIN OWA WEIGHTS}

In this section, we propose a new model to obtain the OWA weights base on normal distribution function as follow:

$$
\left\{\begin{array}{c}
\min J_{1}=\sum_{i=1}^{n}\left(w_{i}-\tilde{w}_{i}\right)^{2}, \\
\text { s.t. } ? \sum_{i=1}^{n} \frac{n-i}{n-1} w_{i}=\alpha, \\
\sum_{i=1}^{n} w_{i}=1, \\
w_{i} \geq 0, i=1,2, \cdots, n,
\end{array}\right.
$$

where $\quad \tilde{w}_{i}=\frac{\Phi\left(a_{i}\right)-\Phi\left(a_{i-1}\right)}{\Phi\left(a_{n}\right)-\Phi\left(a_{1}\right)}, a_{i}=i-\frac{n}{2}$.

It is easy to prove that $\tilde{w}_{i}$ is strictly monotone increasing in $[1, n / 2]$, and decreasing in $[n / 2, n]$; and $\tilde{w}_{i}$ is symmetrical to the most center element of $\left(\tilde{w}_{1}, \tilde{w}_{2}, \ldots, \tilde{w}_{n}\right)$, that is $\tilde{w}_{i}=\tilde{w}_{n-i}$. Such properties ensure that values in the middle play more important roles than values in the two ends, which is similar to trimmed mean. Given $n=5$, the solution of (3) is shown in Figure 1, from which one can see that the obtained weights are symmetric, and they can reasonably adjusted by the orness level.

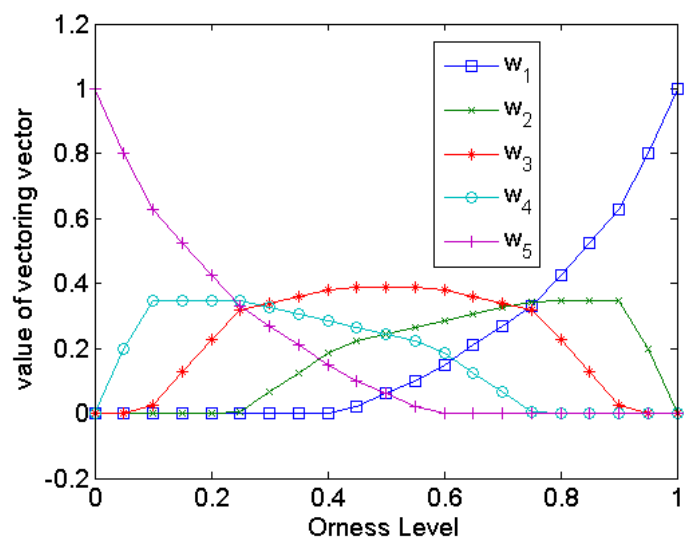

Fig.1. Result of the new model

Below we will prove some properties of the new proposed OWA model:

$$
\text { Theorem1. } \quad W_{\max }=(1,0, \ldots, 0)
$$

$W_{\text {min }}=(0, \ldots, 0,1)$ are the optimal solutions of model (3) if and only if orness level $\alpha=1$ and $\alpha=0$.

\section{Proof: (Sufficient conditions)}

when $W_{\max }=(1,0, \ldots, 0)$,

$$
\alpha=\sum_{i=1}^{n} \frac{n-i}{n-1} w_{i}=\frac{n-1}{n-1} \times 1+0+\cdots+0=1 .
$$

(Necessary conditions) Notice $\sum_{i=1}^{n} w_{i}=1$, we have

$$
\begin{gathered}
\alpha=\sum_{i=1}^{n} \frac{n-i}{n-1} w_{i}=\sum_{i=1}^{n}\left(1-\frac{i-1}{n-1}\right) w_{i} \\
=\sum_{i=1}^{n} w_{i}-\sum_{i=1}^{n} \frac{i-1}{n-1} w_{i}=1-\sum_{i=1}^{n} \frac{i-1}{n-1} w_{i}=1
\end{gathered}
$$

then

$\sum_{i=1}^{n} \frac{i-1}{n-1} w_{i}=\frac{1}{n-1} w_{2}+\frac{2}{n-1} w_{3}+\cdots+\frac{n-2}{n-1} w_{n-1}=0$

since $w_{i} \geq 0$, we have

$$
w_{1}=1, w_{2}=w_{3}=\cdots=w_{n}=0 .
$$


Thus we prove $W_{\max }=(1,0, \ldots, 0)$ is the optimal solution if and only if $\alpha=1$. Similarly, we can prove that $W_{\min }=(0, \ldots, 0,1)$ is the optimal solution if and only if $\alpha=0$.

Theorem2. $\tilde{W}=\left(\tilde{w}_{1}, \tilde{w}_{2}, \ldots, \tilde{w}_{n}\right)$ is the optimal solutions of model (3) if and only if orness level $\alpha=1 / 2$.

Proof : (Sufficient conditions) if $n=2 m$ is even, then

$$
\begin{aligned}
\alpha & =\sum_{i=1}^{n} \frac{n-i}{n-1} w_{i}=\sum_{i=1}^{2 m} \frac{n-i}{n-1} \tilde{w}_{i} \\
& =\sum_{i=1}^{m}\left(\frac{n-i}{n-1}+\frac{n-1-(n-i)}{n-1}\right) \tilde{w}_{i} \\
& =\sum_{i=1}^{m} \tilde{w}_{i}=\frac{1}{2}
\end{aligned}
$$

If $n=2 m+1$ is odd, then

$$
\begin{aligned}
\alpha & =\sum_{i=1}^{n} \frac{n-i}{n-1} w_{i}=\sum_{i=1}^{2 m+1} \frac{n-i}{n-1} \tilde{w}_{i} \\
& =\sum_{i=1}^{m}\left(\frac{n-i}{n-1}+\frac{n-1-(n-i)}{n-1}\right) \tilde{w}_{i}+\frac{n-m-1}{n-1} \tilde{w}_{m+1} \\
& =\sum_{i=1}^{m} \tilde{w}_{i}+\frac{1}{2} \tilde{w}_{m+1}=\frac{1}{2}
\end{aligned}
$$

(Necessary conditions) If the objective function $J_{1}=\sum_{i=1}^{n}\left(w_{i}-\tilde{w}_{i}\right)^{2}$ of model (3) achieves the minimum zero, then $w_{i}$ must equal to $\tilde{w}_{i}$. Therefore, the weighting vector $W$ is equal to $\tilde{W}$. So, $\tilde{W}$ is the optimal solution of model (3).

Theorem3. If $W=\left(w_{1}, w_{2}, \cdots, w_{n}\right)$ is the optimal solutions of model (3) when the value of orness level is $\alpha$, then $w=\left(w_{n}, w_{n-1}, \cdots, w_{1}\right)$ if the optimal solution of (3) when the value of orness level is $1-\alpha$.

Proof: Under a orness level of $\alpha$, if $W=\left(w_{1}, w_{2}, \cdots, w_{n}\right)$ is the optimal solution of (3), then its objective function $J_{1}=\sum_{i=1}^{n}\left(w_{i}-\tilde{w}_{i}\right)^{2}$ achieves the minimum, and its conditions

$\sum_{i=1}^{n} \frac{n-i}{n-1} w_{i}=\alpha, \quad \sum_{i=1}^{n} w_{i}=1$, holds. Now we will show that $w=\left(w_{n}, w_{n-1}, \cdots, w_{1}\right)$ is the optimal solution of

$$
\left\{\begin{array}{l}
\min J_{2}=\sum_{i=1}^{n}\left(w_{i}-\tilde{w}_{i}\right)^{2}, \\
\sum_{i=1}^{n} \frac{n-i}{n-1} w_{i}=1-\alpha, \\
\sum_{i=1}^{n} w_{i}=1, \quad w_{i} \geq 0, i=1,2, \cdots, n,
\end{array}\right.
$$

For one thing, since $\sum_{i=1}^{n} w_{n+1-i}=1$

and $\sum_{i=1}^{n} \frac{n-i}{n-1} w_{n+1-i}=1-\sum_{i=1}^{n} \frac{i-1}{n-1} w_{n+1-i}=1-\alpha$

$w=\left(w_{n}, w_{n-1}, \cdots, w_{1}\right)$ is a feasible solution. For another, because $\tilde{w}_{i}=\tilde{w}_{n-i}, \quad J_{2}=\sum_{i=1}^{n}\left(w_{n+1-i}-\tilde{w}_{i}\right)^{2}$ is always equal to $J_{1}$, the objective function of (3). Since $J_{1}$ achieves minimum at $\left(w_{1}, w_{2}, \cdots, w_{n}\right)$, $J_{2}$ achieves minimum at $\left(w_{n}, w_{n-1}, \cdots, w_{1}\right)$. That is to say, $\left(w_{n}, w_{n-1}, \cdots, w_{1}\right)$ is the optimal solution of the model with an orness level of $1-\alpha$.

\section{EXPERIMENT}

27 red wines are to be assessed by a wine commission that consists of 10 professional tasters. Each taster is require to assess appearance, color, flavor, taste, and overall impression of each wine. Total scores of these assessment are listed in Table 1.

Tab.1. scores of 22 red wines from 10 judgers

\begin{tabular}{|l|l|l|l|l|l|l|l|l|l|l|}
\hline & $\mathrm{J} 1$ & $\mathrm{~J} 2$ & $\mathrm{~J} 3$ & $\mathrm{~J} 4$ & $\mathrm{~J} 5$ & $\mathrm{~J} 6$ & $\mathrm{~J} 7$ & $\mathrm{~J} 8$ & $\mathrm{~J} 9$ & $\mathrm{~J} 10$ \\
\hline wine1 & 71 & 81 & 86 & 74 & 91 & 80 & 83 & 79 & 85 & 73 \\
\hline wine2 & 80 & 85 & 89 & 76 & 69 & 89 & 73 & 83 & 84 & 76 \\
\hline wine3 & 52 & 64 & 65 & 66 & 58 & 82 & 76 & 63 & 83 & 77 \\
\hline wine4 & 74 & 74 & 72 & 62 & 84 & 63 & 68 & 84 & 81 & 71 \\
\hline wine5 & 72 & 69 & 71 & 61 & 82 & 69 & 69 & 64 & 81 & 84 \\
\hline wine6 & 63 & 70 & 76 & 64 & 59 & 84 & 72 & 59 & 84 & 84 \\
\hline wine7 & 64 & 76 & 65 & 65 & 76 & 72 & 69 & 85 & 75 & 76 \\
\hline wine8 & 77 & 78 & 76 & 82 & 85 & 90 & 76 & 92 & 80 & 79 \\
\hline wine9 & 67 & 82 & 83 & 68 & 75 & 73 & 75 & 68 & 76 & 75 \\
\hline wine10 & 73 & 60 & 72 & 63 & 63 & 71 & 70 & 66 & 90 & 73 \\
\hline wine11 & 69 & 84 & 79 & 59 & 73 & 77 & 77 & 76 & 75 & 77 \\
\hline wine12 & 70 & 77 & 70 & 70 & 80 & 59 & 76 & 76 & 76 & 76 \\
\hline wine13 & 72 & 80 & 80 & 71 & 69 & 71 & 80 & 74 & 78 & 74 \\
\hline wine14 & 70 & 79 & 91 & 68 & 97 & 82 & 69 & 80 & 81 & 76 \\
\hline wine15 & 76 & 84 & 84 & 66 & 68 & 87 & 80 & 78 & 82 & 81 \\
\hline wine16 & 78 & 84 & 76 & 71 & 82 & 79 & 76 & 76 & 86 & 81 \\
\hline wine17 & 73 & 90 & 96 & 71 & 69 & 60 & 79 & 73 & 86 & 74 \\
\hline wine18 & 73 & 83 & 72 & 68 & 93 & 72 & 75 & 77 & 79 & 80 \\
\hline wine19 & 70 & 85 & 90 & 68 & 90 & 84 & 70 & 75 & 78 & 70 \\
\hline wine20 & 60 & 78 & 81 & 62 & 70 & 67 & 64 & 62 & 81 & 67 \\
\hline wine21 & 73 & 80 & 71 & 61 & 78 & 71 & 72 & 76 & 79 & 77 \\
\hline wine22 & 70 & 77 & 63 & 64 & 80 & 76 & 73 & 67 & 85 & 75 \\
\hline
\end{tabular}

Source: these data are selected from 2012 Chinese mathematical constest problems. (http://www.mcm.edu.cn/problem/2012/2012.html) 
Set $\alpha$ be $0.1,0.3,0.5,0.7$ and 0.9 respectively, and use model (3) to calculate the weighting vectors. The OWA assessments of 27 wines under different orness levels are listed in Table 2, from which one can see that for an optimistic evaluator(when $\alpha$ is 0.7 or 0.9 ), No.14 is viewed as the best, because the highest score, 97, from No.5 judger plays an important role in the assessment for an optimistic evaluator. No. 2 wine is reviewed as the best in a neutral evaluator's eyes(when $\alpha$ is 0.5), because it receives fairly good scores from most of the judgers. Though No.8 wine receives no scores higher than 90 from the 10 judgers, it is still regarded as the best wine for a pessimistic evaluator(when $\alpha$ is 0.1 or 0.3 ), because each judger give it a not-too-bad score, which is higher than 76.

Tab.2. OWA assessments under different orness levels

\begin{tabular}{|c|c|c|c|c|c|}
\hline$\alpha$ & 0.9 & 0.7 & 0.5 & 0.3 & 0.1 \\
\hline wine1 & 87.87 & 84.06 & 80.59 & 76.84 & 72.83 \\
\hline wine2 & 87.95 & 84.71 & $81.05^{*}$ & 76.60 & 71.97 \\
\hline wine3 & 80.69 & 73.52 & 66.93 & $61.36^{* *}$ & $56.38 * *$ \\
\hline wine4 & 82.62 & 77.52 & 72.93 & 68.73 & 64.00 \\
\hline wine5 & 81.77 & 75.63 & 70.36 & 67.12 & 63.67 \\
\hline wine6 & 82.95 & 77.11 & 70.84 & 65.38 & 60.34 \\
\hline wine7 & 80.29 & 76.77 & 73.09 & 69.09 & 65.10 \\
\hline wine8 & 89.43 & 84.21 & 79.71 & $77.90 *$ & $76.38 *$ \\
\hline wine9 & 81.04 & 77.62 & 74.60 & 71.63 & 68.14 \\
\hline wine10 & 81.06 & 75.18 & 69.98 & 66.17 & 62.09 \\
\hline wine11 & 80.97 & 78.48 & 76.29 & 71.48 & 65.32 \\
\hline wine12 & $78.23^{* *}$ & 76.94 & 75.05 & 70.53 & 65.03 \\
\hline wine13 & 79.56 & 77.01 & 74.34 & 72.10 & 70.25 \\
\hline wine14 & $91.73 *$ & $84.97 *$ & 79.08 & 74.53 & 69.53 \\
\hline wine15 & 85.19 & 82.78 & 80.33 & 75.21 & 69.14 \\
\hline wine16 & 84.30 & 81.38 & 78.53 & 76.10 & 73.70 \\
\hline wine17 & 91.00 & 81.97 & 74.41 & 69.89 & 65.30 \\
\hline wine18 & 86.95 & 81.12 & 76.00 & 73.08 & 70.28 \\
\hline wine19 & 88.43 & 82.76 & 76.69 & 72.46 & 69.32 \\
\hline wine20 & 79.34 & 72.96 & $67.14 * *$ & 64.14 & 61.41 \\
\hline wine21 & 79.10 & 76.91 & 74.50 & 70.61 & 66.32 \\
\hline wine22 & 81.59 & 77.58 & 73.64 & 69.31 & 64.67 \\
\hline
\end{tabular}

* maximum score

** minimum score

\section{CONCLUSIONS}

Based on the good properties of normal distribution function, a new method to generate a weighting vector for OWA assessment is proposed. Our model is a quadratic programming problem. Similar to the well-known trimmed mean, such weighting vector emphasizes the values in the middle place, and three properties about such weighting vector, which is the solution of our model, is proofed. These properties show that the parameter of our model, namely, the orness level, can represent the degree of optimism of an evaluator. As an example, we use these weighting vectors to assess the qualities of different wines, under different orness levels. The result of this example is consistent with subjective sensations of different people with different degrees of optimism.

\section{ACKNOWLEDGEMENTS}

This project is sponsored by the Science Foundation of Fujian Education Department (JA12273, JK2013037), and Science Foundation of Science and Technology Bureau of Quanzhou (2012Z103). Corresponding author: Yang Xiyang, yangxiyang@ 139.com.

\section{REFERENCES}

[1] Yager R R. Families OWA operators. Fuzzy sets and systems, 1993, 59: 125-148

[2] Valentina Ferretti, Silvia Pomarico. Ecological land suitability analysis through spatial indicators: An application of the Analytic Network Process technique and Ordered Weighted Average approach. Ecological Indicators, Volume 34 (2013): 507-519

[3] Pece V. Gorsevski, Katerina R. Donevska, Cvetko D. Mitrovski, Joseph P. Frizado. Integrating multi-criteria evaluation techniques with geographic information systems for landfill site selection: A case study using ordered weighted average. Waste Management, Volume 32, Issue 2, (2012): 287-296

[4] M. O'Hagan, Aggregating template rule antecedents in real-time expert systems with fuzzy set logic, in: Proceedings of the 22nd Annual IEEE Asilomar Conference on Signals, Systems, Computers, Pacific Grov, CA, (1988): 681-689.

[5] R. Fulle'r, P. Majlender, An analytic approach for obtaining maximal entropy OWA operator weights, Fuzzy Sets and Systems 124 (2001): 53-57.

[6] R. Fuller, P.Majlender. On obtaining minimal variability OWA operator weights. Fuzzy Sets Syst. 136 (2003): 203215

[7] Y. Wang, Y. Luo, X. Liu, Two new models for determining OWA operator weights, Comput. Ind. Eng. 52 (2007): 203-209.

[8] Y. M. Wang, C. Parkan, A preemptive goal programming method for aggregating OWA operator weights in group decision making, Information Sciences 177 (2007): 18671877.

[9] X. Liu, The solution equivalence of minimax disparity and minimum variance problems for OWA operators, International Journal of Approximate Reasoning, 2007, 45 (1): 68-81.

[10] Wang Yu, Xu Zeshui. A new Method of giving OWA weights. mathematics in practice and theory, 2008 (03): 51-61

[11] Yang Xiyang, Xiao Xiaoyu, Li Zhiwei. A new method to obtain the OWA weights based on combination numbers. Journal of Hunan University of Science \& Technology: Natural Science Editon, 2014, 29 (3): 125-128. 